\title{
CONSTRUCCIÓN DE UNA PRUEBA DE INTELIGENCIA EMOCIONAL
}

\author{
Luis M. Escurra Mayaute \\ Ana E. Delgado Vásquez. \\ María Rosario Quesada Murillo \\ José C. Rivera Benavides \\ Julio Santos Islas \\ Gerardo Rivas Castro \\ Juan Pequeña Constantino
}

\section{RESUMEN}

Se ha construido una prueba para medir la Inteligencia emocional tomando como base la propuesta de Salovey y Mayer. Se construyeron items pertenecientes a los siguientes cinco dominios: a) Autoconocimiento emocional, b) Control Emocional, c) Automotivación, d) Empatía y e) Habilidad paro las relaciones Interpersonales. La prueba se aplicó a una muestra de estudiantes del primer semestre de las diversas especialidades de la U.N.M.S.M. Los cuales corresponden a una muestra aleatoria de 489 alumnos, pertenecientes de manera representativa a las 19 Facultades de las 5 áreas de especialización. El análisis Psicométrico de los ítems permitió eliminar aquellos que no eran significativos, la prueba presentó Confiabilidad por consistencia interna, a través del coeficiente Alfa de Cronbach tanto en general como en los dominios evaluados. También se observó que el instrumento presentó Validez de Contenido y Validez de Constructo.

Palabras clave: Inteligencia Emocional, Análisis Psicométrico, Confiabilidad, Validez.

\begin{abstract}
Under the theoretical framework of Salovey and Moyer a psychometric test has been created to measure Emotional Intelligence. Five domains have been considered for items elaboration: a) Emotional Selfknowledge; b) Emotional Control; c) Self motivation; d) Empathy and e) Interpersonal relations. A randomly sample of 489 first level students was selected from 19 different University Programs which belong to five Academic Areas. The psychometric item analysis permited to take not statistically significant items out .. The Test reported internal consistency reliability through Alfa Cronbach Coeficient. It has been reported for each domain evaluated and for the general test. It has been also confirmed content and construct validity for the test.
\end{abstract}

Key words: Emotional Inteligence / Psycometric analysis / Reliabilily / Validity. 


\section{INTRODUCCIÓN}

La Inteligencia Emocional tiene sus raíces en el concepto de «inteligencia social,» inicialmente identificado por Thorndike en 1920. Otros autores han identificado otro tipo de inteligencias agrupándolas de la siguiente manera:

a) Inteligencia Abstracta, que es la habilidad de entender y manipular símbolos verbales y matemáticos.

b) Inteligencia Concreta, que es la habilidad de entender y manipular con objetos e

c) Inteligencia Social, que es la habilidad de entender y relacionar a las personas (Ruisel, 1992).

La inteligencia social ha sido definida como la habilidad de entender y manejar a los hombres y mujeres, muchachos y muchachas para actuar sabiamente en relaciones humanas.

Gardner (1983) incluye las inteligencias Intrapersonal e Interpersonal en su teoría de las inteligencias múltiple. Estos dos inteligencias serían las que conforman la inteligencia social y las define de la siguiente forma:

a. Inteligencia interpersonal es la habilidad de entender a otras personas, identificar lo que los motiva, cómo trabajan, cómo trabajar cooperativamente con ellos.

b. Inteligencia Intrapersonal, es la habilidad para reconocer los aspectos internos de si mismo, el acceso a la propia vida emocional, a la propia gama de sentimientos, la capacidad de efectuar discriminaciones entre emociones y ponerles un nombre y recurrir a ellas como un medio de interpretar y orientar la propia conducta.

Según Salovey \& Mayer (1990), la inteligencia emocional, incluye la inteligencia intrapersonal y la inteligencia interpersonal. así como otras habilidades que pueden categorizarse en 5 dominios:

a) Conocimiento de si mismo, observándose y reconociendo un sentimiento cuando pasa.

b) Control Emocional, implica el manejo de las emociones de forma que sean apropiados; comprendiendo lo que está detrás de un sentimiento; encontrando maneras de manejar miedos y ansiedades, encolerice, y tristeza.

c) Automotivación, es la capacidad de Motivarse a sí mismo, encauzando las emociones en el servicio de una meta.

d) Empatía, es el reconocimiento de las emociones ajenas, es la sensibilidad para percibir los sentimientos y preocupaciones de los otros, apreciando las diferencias que se presentan en la forma cómo las personas se sienten sobre las cosas.

e) Habilidad para las relaciones Interpersonales, es el control de la emociones en los otros; incluye la competencia social y las habilidades sociales.

El conocimiento de si mismo (inteligencia del intrapersonal), la empatía y el manejo de las relaciones (inteligencia interpersonal) son esencialmente dimensiones de inteligencia social.

Goleman (1996) presento su definición de inteligencia emocional basándose en los trabajos desarrollados por Mayer y Salovey (1993). Sin embargo agregó muchas cosas que él presentó como inteligencia emocional., como es el caso de la inclusión de variables que podrían considerarse más como «rasgos de personalidad» que componentes de inteligencia 
emocional. Así por ejemplo, incluyó el optimismo, la perseverancia y la habilidad de retardar satisfacción como aspectos relevantes de la inteligencia emocional.

En la actualidad existe discordancia acerca de que la inteligencia emocional es más un potencial inherente. o si es un conjunto de «habilidades» o «competencias». Por ejemplo para Goleman (1997), la Inteligencia emocional es básicamente la misma a lo largo de la vida

Mayer y Salovey (1997) han actualizado su definición de Inteligencia Emocional, indicando que involucra la habilidad de percibir con precisión, estimar, y expresar las emociones; la habilidad de acceder y/o generar sentimientos cuando ellos facilitan el pensamiento; la habilidad de entender emoción y el conocimiento emocional; y la habilidad de regular las emociones para promover crecimiento emocional y intelectual.

El campo de estudio de la Inteligencia Emocional, presenta dos afrontes generales. El primer afronte es el socio-emocional que incluye habilidades así como una serie de rasgos de personalidad. Esta concepción fue popularizada por el libro de Goleman (1996). En alguna magnitud, el modelo de Salovey y Mayer (1990) es también un modelo socioemocional, o mixto.

El segundo afronte corresponde a un modelo de habilidad. Este modelo de inteligencia emocional establece un eslabón entre las emociones y el razonamiento.

El modelo de habilidad de inteligencia emocional, como el desarrollado por Mayer y Salovey (1997), presenta a la inteligencia emocional como un juego de habilidades que combinan emociones y cognición. Ellos definieron la inteligencia emocional como la habilidad de percibir emociones, acceder y generar emociones para ayudar al pensamiento, entender las emociones, aumentar el conocimiento emocional. y regular las emociones reflexivamente para promover el crecimiento emocional e intelectual (Mayer \& Salovey, 1997). Específicamente, la inteligencia emocional esta compuesta por 4 componentes separados:

a. Identificación de Emociones, es la habilidad de reconocer en uno y en los demás como se sienten.

b. Utilización de Emociones, es la habilidad para generar emociones y razonar con esta emoción.

c. Comprensión de las Emociones, es la habilidad para entender las emociones complejas y las «cadenas» de emociones, cómo la transición de emociones de una fase a otra,

d. Dirección de las Emociones, es la habilidad para manejar la emociones en su ego y en otros.

El análisis de la bibliografía especializada a nivel Internacional indica que: los investigadores que estudiaron las dimensiones de inteligencia emocional midieron los conceptos relacionados, como las habilidades sociales, competencia interpersonal, madurez psicológica y conocimiento emocional, antes que se acuñara el término «inteligencia emocional» (Young, 1996). Para Goleman (1996), los maestros de las escuelas primarias americanas, han estado enseñando los rudimentos de inteligencia emocional desde 1978, con el desarrollo del plan de estudios de la ciencia y la enseñanza de clases como el desarrollo social, el aprendizaje social y emocional, y la inteligencia personal, todos los cuales apuntaron al nivel de competencia social y emocional (Goleman, 1997). También se han real izado diferentes investigaciones que reportan el estudio de las relaciones entre la Inteligencia Emocional y otros constructos psicológicos como es el caso del Liderazgo 
(Ashforth \& Humphrey. 1995), el rendimiento grupal (Williams \& Sternberg, 1988), el rendimiento individual, las relaciones interpersonales y sociales, el intercambio social, el manejo del cambio y la evaluación de los rendimientos (Goleman, 1996).

En lo que concierne a la revisión de la literatura especializada sobre el tema en el ámbito nacional, se ha podido establecer que no existen estudios relacionados con el tema de estudio. En la actualidad en el campo de la metodología de investigación psicológica ha logrado notoriedad la propuesta de Marín (1986), quien indica la necesidad de distinguir entre los constructos Éticos (universales) y los Emicos (particulares), en lo que se refiere al desarrollo de las investigaciones en América Latina.

Por otro lado al momento de plantearse la investigación no existen investigaciones nacionales sobre el tema de la Inteligencia Emocional, ni tampoco instrumento alguno que permita medir dicho constructo, notándose que las pruebas existentes provienen de otros contextos socioculturales diferentes al nuestro. Es sobre la base de estas consideraciones que nos proponemos estudiar sí: ¿la prueba construida para medir la Inteligencia Emocional presenta validez y confiabilidad?

\section{Justificación}

La inteligencia Emocional es un constructo que está en pleno desarrollo en la psicología contemporánea, motivo por el cual no ha sido estudiado de forma empírica en nuestro medio.

Con la finalidad de impulsar el desarrollo en nuestro medio de estudios acerca de este tipo de inteligencia es indispensable desarrollar que pueda medir dicho constructo de forma válida y confiable para nuestro medio, de forma que se pueda llegar a generar un conocimiento más exacto del tema, que permita el desarrollo de aplicaciones prácticas en e los diferentes campos del quehacer psicológico.

\section{Objetivos}

\section{a. Generales}

Desarrollar una prueba que mida la Inteligencia Emocional.

\section{b. Específicos}

1. Construir una prueba para medir la Inteligencia Emocional

2. Estudiar la validez de la prueba de Inteligencia Emocional.

3. Estudiar la Confiabilidad de la prueba de Inteligencia Emocional.

4. Desarrollar Baremos provisionales para la prueba de Inteligencia Emocional.

\section{METODO}

El presente trabajo siguiendo a Kerlinger (1988) constituye una investigación de Tipo Metodológico, en la medida que se trata de construir una prueba psicológica que permita medir la inteligencia emocional. Siguiendo a Sánchez y Reyes (1998), la investigación también puede ser clasificada como aplicada, en la media que se orienta a la aplicación de manera práctica de los conocimientos teóricos de un determinado tema. 


\section{Diseño de Investigación.}

El diseño de la investigación corresponde al Correlacional en la medida que los resultados obtenidos en la medición del fenómeno serán utilizados para analizar las características psicométricas del instrumento utilizado (Alarcón. 1991; Hernández, Fernández y Baptista. 1998; Sánchez y Reyes, 1998).

\section{Instrumento de Recolección de Datos.}

En la investigación se ha construido una prueba para medir la inteligencia emocional la cual fue realizada a través de las siguientes etapas sugeridas por Schwab (1998).

a. Desarrollo de la estructura conceptual del Instrumento, sobre la base de los siguientes pasos:

a.1 Se especificó la finalidad del instrumento, en términos de una definición operacional precisa de aquello que se quiere medir.

a.2 Operacionalización de la prueba, una vez que se determino la estructura conceptual, tomando en cuenta los 5 aspectos básicos de la Inteligencia Emocional propuestos por Meyer y Salovey (1993).

a.3 El formato de la prueba seleccionado es de acuerdo a un escalamiento tipo Likert con 5 puntos de calificación, de papel y lápiz y de aplicación colectiva.

b. Desarrollo del formato de la Prueba Inicial. Los ítems fueron elaborados de acuerdo a la estructura conceptual, formulándose todos aquellos que fueron sometidos a un proceso de validez de contenido por criterio de jueces Adicionalmente e incluyeron Ítems que median otros aspectos con la finalidad de evaluar otros aspectos con la finalidad de servir de elementos de distracción y para evaluar otros constructos con la finalidad de realizar posteriores validaciones.

c. Aplicación y Calibración de los resultados de la Prueba Inicial. En ésta fase, la prueba fue aplicada a la muestra de investigación, efectuándose el proceso de análisis de Ítems en base al cálculo de los Promedio y las correlaciones item-Area corregida de cada uno de los Ítems.

d. Elaboración del formato final de la prueba. Con los resultados finales se confeccionó el formato final de la Prueba.

e. Estudio de la validez de Construcción. Se desarrollo el análisis factorial exploratorio en base a los 5 aspectos evaluados, factores, el cual fue ejecutado con el método de factorización de los componentes principales y el método de rotación oblícua, Oblimin.

f. Estudio de la Confiabilidad. Se estudió la consistencia interna utilizando el coeficiente Alfa de Cronbach para cada uno de los aspectos evaluados.

g. Construcción de Baremos provisionales. Los cuales fueron construidos de acuerdo a la transformación en rangos percentiles.

\section{RESULTADOS}

La Prueba de Inteligencia Emocional, mide la habilidad de percibir con precisión, estimar, y expresar las emociones; la habilidad de acceder y/o generar sentimientos cuando ellos 
facilitan el pensamiento; la habilidad de entender emoción y el conocimiento emocional; y la habilidad de regular las emociones para promover crecimiento emocional y intelectual. La prueba evalúa los siguientes aspectos:

a) Autoconocimiento Emocional, mide la habilidad para reconocer sus propios sentimiento, pudiendo diferenciarlos entre sí.

b) Control Emocional, mide la habilidad para controlar los sentimientos; comprendiendo lo que está detrás de ellos, así como las maneras de manejar los miedos, ansiedades, cóleras y tristezas.

c) Automotivación, mide la habilidad del sujeto para motivarse a sí mismo y encausar sus emociones en el servicio de una meta, de manera que el control emocional; permite posponer la satisfacción de los impulsos.

d) Empatía (Reconocimiento de las emociones Ajenas), mide la habilidad para reconocer las emociones ajenas, es decir la sensibilidad para asumir los sentimientos y preocupaciones que presentan los demás.

e) Habilidad para las relaciones Interpersonales, mide la habilidad para dirigir las emociones, la competencia social y las habilidades sociales.

La muestra de sujetos con la que se trabajó ascendió a 489 casos, distribuidos de forma proporcional según las áreas y E. A. P. De las distintas facultades de la universidad (ver Tabla No. 1).

Tabla No. 1-A

COMPOSICIÓN DE LA POBLACIÓN Y MUESTRA

\begin{tabular}{|l|c|c|}
\hline \multicolumn{1}{|c|}{ E.A.P. } & $\mathbf{P .}$ & $\mathbf{M .}$ \\
\hline QUIMICA & 89 & 8 \\
CIENCIAS BIOLOGÍCAS & 137 & 12 \\
FISICA & 84 & 7 \\
MATEMATICAS & 84 & 7 \\
ESTADISTICA & 116 & 10 \\
INVESTIGAC. OPERATIVA & 137 & 12 \\
TOTAL AREA A & $\mathbf{6 4 7}$ & $\mathbf{5 7}$ \\
\hline MEDICINA & 189 & 17 \\
FARMACIA & 84 & 7 \\
ODONTOLOGIA & 86 & 8 \\
VETERINARIA & 88 & 8 \\
OBSTETRICIA & 116 & 10 \\
ENFERMERIA & 137 & 12 \\
LAB. CLINICO y PATOL. & 68 & 6 \\
TERAPIA FISICA & 37 & 3 \\
RADIOLOGIA & 37 & 3 \\
TERAPIA OCUPACIONAL & 37 & 3 \\
NUTRICION & 80 & 7 \\
PSÍCOLOGIA & 189 & 17 \\
TOTAL AREA B & $\mathbf{1 1 4 8}$ & $\mathbf{1 0 2}$ \\
\hline MECANICA DE FLUIDOS & 105 & 9 \\
GEOLOGICA & 47 & 4 \\
GEOGRAFICA & $\mathbf{7 9}$ & 7 \\
ING. DE MINAS & 57 & 5 \\
ING. METALURGICA & 63 & 6 \\
ING. INDUSTRIAL & 210 & 19 \\
ING. ELECTRONICA & 141 & 13 \\
ING. QUIMICA & 126 & 11 \\
ING. DE SISTEMAS & 216 & 19 \\
ING. ELECTRICA & $\mathbf{7 5}$ & 7 \\
TOTAL AREA C & $\mathbf{1 1 1 9}$ & $\mathbf{1 0 0}$ \\
\hline & & \\
\hline
\end{tabular}


Tabla No. 1-B

COMPOSICIÓN DE LA POBLACIÓN Y MUESTRA

\begin{tabular}{|l|c|c|}
\hline \multicolumn{1}{|c|}{ E.A.P. } & P. & M. \\
\hline CONTABILIDAD & 368 & 33 \\
ECONOMIA & 312 & 28 \\
ADMINISTRACIÓ & 253 & 22 \\
TURISMO & 126 & 10 \\
NEGOCIOS INTER AC. & 127 & 12 \\
TOTAL AREA D & $\mathbf{1 1 8 6}$ & $\mathbf{1 0 5}$ \\
\hline DERECHO & 315 & 28 \\
FILOSOFIA & 63 & 6 \\
LINGÜISTICA & 63 & 6 \\
COMUNICACIÓN SOCIAL & 95 & 8 \\
ARTE & 53 & 5 \\
BIBLIOTECOLOGIA & 63 & 6 \\
HISTORIA & 72 & 6 \\
SOCIOLOGIA & 72 & 6 \\
ANTROPOLOGIA & 72 & 6 \\
ARQUEO LOGIA & 66 & 6 \\
TRABAJO SOCIAL & 91 & 8 \\
LITERATURA & 63 & 6 \\
EDUCACION & 158 & 14 \\
EDUCUCACION FISICA & 105 & 9 \\
GEOGRAFIA & 50 & 4 \\
TOTAL AREA E & $\mathbf{1 4 0 1}$ & $\mathbf{1 2 5}$ \\
\hline TOTAL & $\mathbf{5 5 0 1}$ & $\mathbf{4 8 9}$ \\
\hline
\end{tabular}

Tabla No. 2

VALIDEZ DE CONTENIDO POR CRITERIO DE JUECES

\begin{tabular}{|c|c|}
\hline Item & V Aiken \\
\hline $\mathbf{0 1}$ & \\
$\mathbf{0 2}$ & $0.80 *$ \\
$\mathbf{0 3}$ & $0.80 *$ \\
$\mathbf{0 4}$ & $1.00 *$ \\
$\mathbf{0 5}$ & $0.80 *$ \\
$\mathbf{0 6}$ & $0.80 *$ \\
$\mathbf{7 9}$ & $0.90 *$ \\
$\mathbf{1 0 2}$ & $0.80 *$ \\
$\mathbf{1 0 6}$ & $1.00 *$ \\
& $0.80 *$ \\
\hline
\end{tabular}

Autoconocimiento Emocional

\begin{tabular}{|c|c|}
\hline Item & V Aiken \\
\hline $\mathbf{0 8}$ & $0.90^{*}$ \\
$\mathbf{2 0}$ & $0.80^{*}$ \\
$\mathbf{2 1}$ & $1.00^{*}$ \\
$\mathbf{2 2}$ & $0.80^{*}$ \\
$\mathbf{3 1}$ & $0.80^{*}$ \\
$\mathbf{3 2}$ & $0.90^{*}$ \\
$\mathbf{3 3}$ & $0.80^{*}$ \\
$\mathbf{3 4}$ & $1.00^{*}$ \\
$\mathbf{3 5}$ & $1.00^{*}$ \\
$\mathbf{3 6}$ & $0.80^{*}$ \\
$\mathbf{4 2}$ & $0.80^{*}$ \\
$\mathbf{4 5}$ & $1.00^{*}$ \\
$\mathbf{4 7}$ & $0.80^{*}$ \\
$\mathbf{5 2}$ & $0.80^{*}$ \\
$\mathbf{6 3}$ & $0.90^{*}$ \\
$\mathbf{7 1}$ & $0.80^{*}$ \\
$\mathbf{7 2}$ & $1.00^{*}$ \\
$\mathbf{8 3}$ & $0.80^{*}$ \\
$\mathbf{8 4}$ & $0.90 *$ \\
& \\
\hline
\end{tabular}

Control Emocional

$* p<.05$

Jueces $=10$

\begin{tabular}{|c|c|}
\hline Item & V Aiken \\
\hline & \\
$\mathbf{1 3}$ & $0.80^{*}$ \\
$\mathbf{1 9}$ & $0.80^{*}$ \\
$\mathbf{2 3}$ & $1.00^{*}$ \\
$\mathbf{2 4}$ & $0.80^{*}$ \\
$\mathbf{2 5}$ & $0.80^{*}$ \\
$\mathbf{2 7}$ & $0.90^{*}$ \\
$\mathbf{2 8}$ & $0.80^{*}$ \\
$\mathbf{3 0}$ & $1.00^{*}$ \\
$\mathbf{3 9}$ & $0.80^{*}$ \\
$\mathbf{4 0}$ & $0.80^{*}$ \\
$\mathbf{4 6}$ & $0.90^{*}$ \\
$\mathbf{5 3}$ & $1.00^{*}$ \\
$\mathbf{5 4}$ & $0.80^{*}$ \\
$\mathbf{5 5}$ & $0.80^{*}$ \\
$\mathbf{5 6}$ & $1.00^{*}$ \\
$\mathbf{5 7}$ & $0.80^{*}$ \\
$\mathbf{5 8}$ & $0.80^{*}$ \\
$\mathbf{5 9}$ & $0.90^{*}$ \\
$\mathbf{6 0}$ & $0.80^{*}$ \\
$\mathbf{6 4}$ & $1.00^{*}$ \\
$\mathbf{6 5}$ & $1.00^{*}$ \\
$\mathbf{7 3}$ & $0.80^{*}$ \\
$\mathbf{7 4}$ & $0.80^{*}$ \\
$\mathbf{7 5}$ & $1.00^{*}$ \\
$\mathbf{7 6}$ & $0.80^{*}$ \\
$\mathbf{7 7}$ & $0.80^{*}$ \\
$\mathbf{7 8}$ & $0.90^{*}$ \\
$\mathbf{8 1}$ & $0.80^{*}$ \\
$\mathbf{8 2}$ & $1.00^{*}$ \\
& \\
\hline
\end{tabular}

Automotivación 
Los resultados de la validez de contenido presentados en las tablas 2 y 3 indican que todos los ítems presentados a los jueces fueron aceptados como representativos del universo de contenido de la Inteligencia Emocional, pues los coeficientes V de Aiken fueron significativos a una $\mathrm{p}<.05$, lo que garantiza que la prueba presenta Validez de Contenido.

Tabla No. 3

VALIDEZ DE CONTENIDO POR CRITERIO DE JUECES

Empatía

(Reconocimiento de las Emociones Ajenas)

\begin{tabular}{|c|c|}
\hline Item & V Aiken \\
\hline 11 & $0.80^{*}$ \\
16 & $0.90^{*}$ \\
18 & $0.80^{*}$ \\
41 & $0.90^{*}$ \\
49 & $1.00^{*}$ \\
50 & $0.80^{*}$ \\
51 & $1.00^{*}$ \\
66 & $0.90^{*}$ \\
\hline
\end{tabular}

Habilidad Para Las Relaciones

interpersonales

\begin{tabular}{|c|c|}
\hline Item & V Aiken \\
\hline 07 & $1.00^{*}$ \\
09 & $0.80^{*}$ \\
10 & $1.00^{*}$ \\
12 & $0.80^{*}$ \\
14 & $0.80^{*}$ \\
15 & $0.90^{*}$ \\
17 & $0.80^{*}$ \\
26 & $1.00^{*}$ \\
29 & $1.00^{*}$ \\
37 & $0.80^{*}$ \\
38 & $0.80^{*}$ \\
43 & $1.00^{*}$ \\
44 & $1.00^{*}$ \\
48 & $0.80^{*}$ \\
61 & $0.90^{*}$ \\
62 & $0.80^{*}$ \\
67 & $1.00^{*}$ \\
68 & $0.80^{*}$ \\
69 & $0.90^{*}$ \\
70 & $0.80^{*}$ \\
80 & $0.90^{*}$ \\
101 & $1.00^{*}$ \\
103 & $0.80^{*}$ \\
104 & $1.00^{*}$ \\
105 & $0.90^{*}$ \\
\hline
\end{tabular}

El análisis cuantitativo de los ítems de cada una de las áreas presentados en las tablas $4,5,6,7$, y 8 , permiten observar que en todos los casos fue necesario realizar dos análisis estadísticos completos para poder calcular la confiabilidad final, debido a que existían ítems que no funcionaron de manera adecuada en la muestra de estudio.

Tabla No. 4

ANÁLISIS DE ITEMS DEL AREA DE AUTOCONOCIMIENTO EMOCIONAL

\begin{tabular}{|c|c|c|c|}
\hline \multicolumn{1}{|c}{ Item } & Media & \multicolumn{1}{c|}{ Analisis 1 ritc } & Analisis 2 ritc \\
\hline 1 & 3.52 & $0,53^{*}$ & $0,61^{*}$ \\
2 & 3.6 & $0,45^{*}$ & $0,55^{*}$ \\
3 & 3.82 & $0,38^{*}$ & $0,45^{*}$ \\
4 & 3.09 & 0.09 & \\
5 & 3.2 & 0.06 & \\
6 & 3.1 & $0,46^{*}$ & $0,59^{*}$ \\
79 & 3.4 & $0,37^{*}$ & $0,41^{*}$ \\
102 & 3.68 & $0,27^{*}$ & $0,30^{*}$ \\
106 & 3.46 & $0,40^{*}$ & $0,45^{*}$ \\
\hline Alfa & \multicolumn{3}{|c}{$0,69^{*}$} \\
$* \mathrm{p}<, 05$ & \multicolumn{3}{c}{$0,81^{*}$} \\
$\mathrm{n}=489$ & \multicolumn{3}{c}{} \\
\end{tabular}


Los resultados de la confiabilidad calculados con el coeficiente Alfa dc Cronbach, permite notar que alcanzaron valores entre 0.81 y 0.87 , lo que nos permite concluir que las áreas de la prueba son confiables.

El análisis de la confiabilidad generalizada de la prueba presentado en la tabla 9 permite establecer que la confiabilidad general de la prueba asciende a 0.90 , resultado que corrobora los hallazgos previos.

Tabla No.5

ANÁLISIS DE ITEMS DEL AREA DE CONTROL EMOCIONAL

\begin{tabular}{|c|c|c|c|}
\hline Item & Media & $\begin{array}{l}\text { Analisis } 1 \\
\text { ritc }\end{array}$ & $\begin{array}{l}\text { Analisis } 2 \\
\text { ritc }\end{array}$ \\
\hline 8 & 3.21 & 0.05 & \\
\hline 20 & 3.91 & $0,41 *$ & $0,44^{*}$ \\
\hline 21 & 3.44 & $0,29 *$ & $0,30^{*}$ \\
\hline 22 & 3.16 & $0,18^{*}$ & $0,27 *$ \\
\hline 31 & 3.72 & $0,40 *$ & $0,38^{*}$ \\
\hline 32 & 3.54 & $0,42 *$ & $0,42 *$ \\
\hline 33 & 3.46 & $0,36^{*}$ & $0,39 *$ \\
\hline 34 & 3.27 & 0.15 & $0,29 *$ \\
\hline 35 & 3.29 & 0.16 & \\
\hline 36 & 3.07 & $0,26^{*}$ & $0,47^{*}$ \\
\hline 42 & 3.43 & 0.08 & \\
\hline 45 & 3.9 & $0,22 *$ & $0,28 *$ \\
\hline 47 & 2.66 & $0,48 *$ & $0,51 *$ \\
\hline 52 & 3.3 & $0,21 *$ & $0,22 *$ \\
\hline 63 & 3.47 & 0.07 & \\
\hline 71 & 3.6 & 0.12 & $0,37 *$ \\
\hline 72 & 3.67 & $0,32 *$ & $0,30 *$ \\
\hline 83 & 3.2 & $0,24 *$ & $0,38 *$ \\
\hline 84 & 3.4 & $0,37 *$ & $0,40 *$ \\
\hline Alfa & \multicolumn{2}{|c|}{$0,70^{*}$} & $0,81^{*}$ \\
\hline
\end{tabular}

$$
* \mathrm{p}<.05
$$

$\mathrm{n}=489$
Tabla No. 6

ANÁLISIS DE ITEMS DEL AREA DE AUTOMOTIVACIÓN

\begin{tabular}{|c|c|c|c|}
\hline Item & Media & $\begin{array}{l}\text { Analisis } 1 \\
\text { ritc }\end{array}$ & $\begin{array}{c}\text { Analisis } 2 \\
\text { ritc }\end{array}$ \\
\hline 13 & 3.59 & $0,22 *$ & $0,24 *$ \\
\hline 19 & 3.79 & $0,50 *$ & $0,54^{*}$ \\
\hline 23 & 3.95 & $0,42 *$ & $0,47 *$ \\
\hline 24 & 3.86 & $0,47 *$ & $0,52^{*}$ \\
\hline 25 & 3.82 & $0,45^{*}$ & $0,53^{*}$ \\
\hline 27 & 3.79 & $0,22 *$ & $0,22 *$ \\
\hline 28 & 3.13 & 0.1 & \\
\hline 30 & 3.79 & $0,28 *$ & $0,38 *$ \\
\hline 39 & 3.96 & $0,36^{*}$ & $0,41 *$ \\
\hline 40 & 3.2 & $0,46^{*}$ & $0,50 *$ \\
\hline 46 & 3.58 & $0,44^{*}$ & $0,49^{*}$ \\
\hline 53 & 2.27 & 0.08 & \\
\hline 54 & 2.42 & 0.06 & \\
\hline 55 & 4.1 & $0,45^{*}$ & $0,56^{*}$ \\
\hline 56 & 3.5 & $0,53 *$ & $0,54 *$ \\
\hline 57 & 3.12 & $0,31 *$ & $0,38 *$ \\
\hline 58 & 3.86 & $0,39 *$ & $0,37 *$ \\
\hline 59 & 4 & $0,43 *$ & $0,45^{*}$ \\
\hline 60 & 4.17 & $0,57 *$ & $0,60 *$ \\
\hline 64 & 3.69 & $0,38 *$ & $0,46^{*}$ \\
\hline 65 & 2.57 & 0.18 & \\
\hline 73 & 3.61 & 0.17 & \\
\hline 74 & 3.48 & $0,23^{*}$ & $0,22 *$ \\
\hline 75 & 3.45 & $0,26^{*}$ & $0,30^{*}$ \\
\hline 76 & 3.36 & $0,25^{*}$ & $0,21 *$ \\
\hline 77 & 3.67 & $0,37 *$ & $0,44^{*}$ \\
\hline 78 & & $0,31 *$ & $0,26^{*}$ \\
\hline 81 & 3.32 & 0.18 & \\
\hline 82 & 3.45 & $0,40 *$ & $0,45^{*}$ \\
\hline Alfa & \multicolumn{2}{|c|}{$0,76^{*}$} & $0,90 *$ \\
\hline
\end{tabular}


Tabla No.7

ANÁLISIS DE ITEMS DEL AREA DE EMPATIA (RECONOCIMIENTO DE LAS EMOCIONES AJENAS)

\begin{tabular}{|c|c|c|c|}
\hline Item & Media & $\begin{array}{l}\text { Análisis } 1 \\
\text { ritc }\end{array}$ & $\begin{array}{c}\text { Análisis } 2 \\
\text { ritc }\end{array}$ \\
\hline 11 & 3.69 & $0,39 *$ & $0,45^{*}$ \\
\hline 16 & 3.08 & $0,34 *$ & $0,28 *$ \\
\hline 18 & 3.9 & $0,43 *$ & $0,47 *$ \\
\hline 41 & 3.38 & 0.12 & \\
\hline 49 & 3.47 & $0,41 *$ & 0.43 \\
\hline 50 & 3.87 & $0,45^{*}$ & 0.48 \\
\hline 51 & 3.75 & $0,39 *$ & 0.42 \\
\hline 66 & 3.18 & 0.03 & \\
\hline Alfa & & $0,60 *$ & $0,79 *$ \\
\hline
\end{tabular}

$* p<, 05$

$n=489$

Tabla No. 9

ANÁLISIS DE LA CONFIABILIDAD DE LAS AREAS

\begin{tabular}{|l|c|c|}
\hline \multicolumn{1}{|c|}{ Areas } & Media & ritc \\
\hline $\begin{array}{l}\text { Autoconocimiento } \\
\text { Emocional }\end{array}$ & 25.84 & $0,65^{*}$ \\
Control Emocional & 50.02 & $0,77^{*}$ \\
Automotivación & 86.62 & $0,77^{*}$ \\
$\begin{array}{l}\text { Empatía (Reconocimiento } \\
\text { de las emociones ajenas) }\end{array}$ & 21.77 & $0,49^{*}$ \\
$\begin{array}{l}\text { Habilidad para las } \\
\text { Relaciones Interpersonales }\end{array}$ & 59.39 & $0,68^{*}$ \\
\hline Alfa Generalizado $=\mathbf{0 , 9 0 *}$ & & \\
\hline
\end{tabular}

$* p<.05$

$n=489$
Tabla No.8

ANÁLISIS DE ITEMS DEL AREA DE HABILIDAD PARA LAS RELACIONES INTERPERSONALES

\begin{tabular}{|c|c|c|c|}
\hline Item & Media & $\begin{array}{c}\text { Análisis 1 } \\
\text { ritc }\end{array}$ & $\begin{array}{c}\text { Análisis 2 } \\
\text { ritc }\end{array}$ \\
\hline $\mathbf{0 7}$ & 3.87 & $0,45^{*}$ & $0,40^{*}$ \\
$\mathbf{0 9}$ & 3.4 & $0,38^{*}$ & $0,35^{*}$ \\
$\mathbf{1 0}$ & 3.14 & $0,28^{*}$ & $0,23^{*}$ \\
$\mathbf{1 2}$ & 3.25 & $0,23^{*}$ & $0,22^{*}$ \\
$\mathbf{1 4}$ & 3.43 & 0.12 & \\
$\mathbf{1 5}$ & 3.31 & 0.17 & \\
$\mathbf{1 7}$ & 2.38 & $0,20^{*}$ & $0,30^{*}$ \\
$\mathbf{2 6}$ & 3.86 & $0,40^{*}$ & $0,39^{*}$ \\
$\mathbf{2 9}$ & 3.18 & 0.13 & \\
$\mathbf{3 7}$ & 2.96 & $0,26^{*}$ & $0,22^{*}$ \\
$\mathbf{3 8}$ & 3.75 & $0,39^{*}$ & $0,38^{*}$ \\
$\mathbf{4 3}$ & 3 & $0,22^{*}$ & $0,27^{*}$ \\
$\mathbf{4 4}$ & 2.69 & $0,25^{*}$ & $0,36^{*}$ \\
$\mathbf{4 8}$ & 2.8 & $0,26^{*}$ & $0,23^{*}$ \\
$\mathbf{6 1}$ & 2.96 & 0.05 & \\
$\mathbf{6 2}$ & 3.07 & 0.08 & \\
$\mathbf{6 7}$ & 3.56 & $0,30^{*}$ & $0,34^{*}$ \\
$\mathbf{6 8}$ & 2.54 & 0.12 & \\
$\mathbf{6 9}$ & 2.56 & 0.06 & \\
$\mathbf{7 0}$ & 4.16 & $0,40^{*}$ & $0,38^{*}$ \\
$\mathbf{8 0}$ & 4.05 & $0,31^{*}$ & $0,41^{*}$ \\
$\mathbf{1 0 1}$ & 3.46 & 0.11 & \\
$\mathbf{1 0 3}$ & 3.7 & $0,38^{*}$ & $0,26^{*}$ \\
$\mathbf{1 0 4}$ & 3.6 & $0,36^{*}$ & $0,33^{*}$ \\
$\mathbf{1 0 5}$ & 3.92 & $0,44^{*}$ & $0,40^{*}$ \\
\hline $\mathbf{A l f a}$ & & & \\
$\mathbf{1 0}$ & & $\mathbf{0 , 7 6 *}$ & $\mathbf{0 , 9 0 *}$ \\
\hline
\end{tabular}

El análisis de la matriz de las correlaciones entre las áreas y el puntaje total, (ver tabla No. 1) permite observar que las correlaciones de las áreas con la inteligencia Emocional total son positivas, estadísticamente significativas y superiores a 0.80 
Tabla $\mathbf{N}^{\circ} 10$

MATRIZ DE CORRELACIÓN ENTRE LAS AREAS

\begin{tabular}{|l|c|c|c|c|c|}
\hline \multicolumn{1}{|c|}{ AREA } & $\begin{array}{c}\text { Inteligencia } \\
\text { Emocional }\end{array}$ & $\begin{array}{c}\text { Autoconoc. } \\
\text { Emocional }\end{array}$ & $\begin{array}{c}\text { Control } \\
\text { Emocional }\end{array}$ & Automotiv. & Empatía \\
\hline Autoconocimiento Emocional & $0,84 * * *$ & & & & \\
Control Emocional & $0,86^{* * *}$ & $0,60^{* * *}$ & & & \\
Automotivación & $0,91 * * *$ & $0,59 * * *$ & $0,72^{* * *}$ & & \\
$\begin{array}{l}\text { Empatía(Reconocimiento de } \\
\text { las emociones ajenas) }\end{array}$ & $0,80^{* * *}$ & $0,45^{* * *}$ & $0,44^{* * *}$ & $0,49 * * *$ & \\
$\begin{array}{l}\text { Habilidad para las Relaciones } \\
\text { Interpersonales }\end{array}$ & $0,82^{* * *}$ & $0,56^{* * *}$ & $0,60^{* * *}$ & $0,64 * * *$ & $0,30^{* * *}$ \\
\hline
\end{tabular}

$$
\begin{aligned}
& * * p<.01 \\
& * * * p<.001 \\
& n=489
\end{aligned}
$$

El estudio de la Validez de Constructo por medio del Análisis factorial Exploratorio, computado a través del procedimiento de los componentes principales y el método de rotación oblicua Oblimin, en la Tabla No. 11 encontramos que los resultados de los análisis preliminares como son la medida de la Adecuación del Muestreo de Kaiser-Meyer-Olkin y el test de esfericidad de Bartlett indican que es factible realizar el análisis factorial y encontrar resultados significativos, en tanto que se alcanza un solo factor, el cual explica el $72.1 \%$ de la varianza total, lo que corrobora que la prueba de Inteligencia Emocional presenta validez de construcción.

Tabla No. 11

\begin{tabular}{|c|c|c|c|}
\hline Areas & Media & Factor & Comunalidad \\
\hline Autoconocimiento Emocional & 25.84 & 0.87 & 0.81 \\
\hline Control Emocional & 50.02 & 0.86 & 0.79 \\
\hline Automotivación & 86.62 & 0.79 & 0.81 \\
\hline $\begin{array}{l}\text { Empatía(Reconocimiento de las } \\
\text { emociones ajenas) }\end{array}$ & 21.77 & 0.78 & 0.76 \\
\hline $\begin{array}{l}\text { Habilidad para las Relaciones } \\
\text { Interpersonales }\end{array}$ & 59.39 & 0.69 & 0.79 \\
\hline Varianza explicada & & 72,10 & \\
\hline \multicolumn{4}{|c|}{ Medida de adecuación del muestreo de Kaiser-Meyer Olkin = 0,83 } \\
\hline \multicolumn{4}{|c|}{ Test de esfericidad de Bartlett $=1113,65^{* * *}$} \\
\hline
\end{tabular}

VALIDEZ DE CONTRUCCIÓN DE LA PRUEBA DE INTELIGENCIA EMOCIONAL A TRAVÉZ DEL ANÁLISIS FACTORIAL EXPLORATORIO 
Adicionalmente se efectuó el Análisis Factorial Confirmatorio, aplicando el programa Amos 4.0, observándose en el Gráfico 1 y la Tabla12, se encuentra que el modelo propuesto de un solo factor presenta en el test de Bondad de Ajuste Chi Cuadrado (Cmin) un valor de 0.21 , el cual con 2 grados de libertad alcanza una probabilidad de .900 , lo que nos indica que el modelo es adecuado, notándose además que dicho modelo es superior al modelo Saturado y al Modelo Independiente, lo cual es corroborado en los estadísticos de análisis de residuales (RMR) y los análisis complementarios de bondad de Ajuste como son el GFI y el AGFI. Hallazgos que certifican que la prueba presenta validez de Construcción. Adicionalmente en la tabla 13 se presentan los Baremos preliminares del puntaje total y de las áreas de la prueba de Inteligencia Emocional.

\section{ANÁLISIS FACTORIAL CONFIRMATORIO DE LA PRUEBA DE INTELIGENCLA EMOCIONAL, EscurTa 1999}

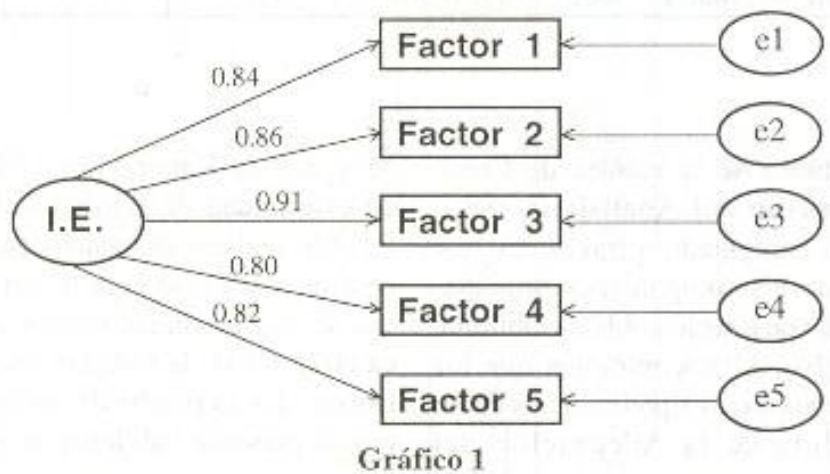

Validez de Construcción a través del Análisis Factorial Confirmatorio

Tabla $\mathbf{N}^{\circ} 12$

VALIDEZ DE CONSTRUCCIÓN DE LA PRUEBA DE INTELIGENCIA EMOCIONAL, RESULTADOS ESTADÍSTICOS DEL ANÁLISIS FACTORAL EXPLORATORIO

\begin{tabular}{|l|r|r|r|}
\hline \multicolumn{1}{|c|}{ Datos } & Modelo & \multicolumn{1}{c|}{$\begin{array}{c}\text { Modelo } \\
\text { Satura. }\end{array}$} & $\begin{array}{r}\text { Modelo } \\
\text { Indepen. }\end{array}$ \\
\hline Parámetros & 13 & 15 & 5 \\
Cmin & 0.21 & 0.00 & 1119.18 \\
G.L. & 2 & 0 & 10 \\
P & 0.900 & & .000 \\
Cmin/G.L. & 0.11 & & 111.92 \\
RMR & 0.07 & 0.00 & 25.42 \\
GFI & 1.00 & 1.00 & 0.46 \\
AGFI & 0.999 & & 0.19 \\
\hline
\end{tabular}




\section{DISCUSION}

Los resultados observados nos indican que en el proceso de construcción de la prueba de inteligencia emocional, fue necesario realizar los análisis varias veces debido a las características particulares que presentan lo sujetos de la muestra, lo cual nos lleva a pensar que en esta clase de instrumentos es necesario realizar también dos procesos de validez de contenido, pues el análisis de los jueces nos permitió establecer que todos los ítems deberían ser incluidos en la versión final de la prueba, pero los resultados empíricos alcanzados nos obligó a eliminar ítems que los jueces aceptaron como válidos.

El proceso de reducción de las áreas a través de los sucesivos análisis nos permitió aproximamos a valores adecuados de confiabilidad, lo que nos permitió alcanzar estimaciones más exactas del constructo evaluado.

Los hallazgos de la validez de construcción trabajados con el análisis Factorial tanto Exploratorio como Confirmatorio. apuntan a corroborar la existencia de un factor común en las cinco áreas analizadas, lo cual confirma lo hipotetizado por la teoría propuesta por diferentes autores como es el caso de Meyer y Salovey (1993) Y por (Goleman (1996), entre otros.

En términos generales los resultados observados nos permiten establecer que la prueba de Inteligencia Emocional es válida y confiable y por lo tanto puede ser utilizada con fines de investigación por los psicólogos interesados en evaluar dicho constructo, pero aún no se recomienda que sea utilizada con fines clínicos, educativos o de diagnóstico, debido a que es necesario realizar otros estudios posteriores de validación que permitan demostrar su efectividad para tomar decisiones.

\section{CONCLUSIONES}

Los resultados de la investigación indican que:

1. La prueba de inteligencia Emocional está conformada por 68 reactivos.

2. La prueba de inteligencia Emocional mide 5 áreas.

3. La prueba de Inteligencia Emocional presenta Validez de contenido.

4. La prueba de Inteligencia Emocional presenta Validez de Construcción, estudiada a través del Análisis Factorial Exploratorio.

5. La prueba de Inteligencia Emocional presenta Validez de Construcción, estudiada a través del Análisis Factorial Confirmatorio.

6. La prueba de Inteligencia Emocional presenta confiabilidad por el método de la Consistencia Interna. 
Tabla $\mathbf{N}^{\circ} 13$

BAREMOS DE LA PRUEBA DE INTELIGENCIA EMOCIONAL

\begin{tabular}{|c|c|c|c|c|c|c|c|}
\hline $\begin{array}{c}\text { Rango } \\
\text { pc }\end{array}$ & Area 1 & Area 2 & Area 3 & Area 4 & Area 5 & $\begin{array}{c}\text { Inteligen. } \\
\text { Emocio. }\end{array}$ & $\begin{array}{c}\text { Rangp } \\
\text { pc. }\end{array}$ \\
\hline $\mathbf{9 9}$ & 34 & 66 & 110 & 30 & 80 & 307 & $\mathbf{9 9}$ \\
$\mathbf{9 5}$ & 33 & 60 & 105 & 28 & 74 & 287 & $\mathbf{9 5}$ \\
$\mathbf{9 0}$ & 32 & 58 & 100 & 26 & 70 & 276 & $\mathbf{9 0}$ \\
$\mathbf{8 5}$ & 31 & 57 & 97 & 25 & 68 & 270 & $\mathbf{8 5}$ \\
\hline $\mathbf{8 0}$ & 30 & 56 & 95 & & 65 & 266 & $\mathbf{8 0}$ \\
$\mathbf{7 5}$ & 29 & 55 & 94 & 24 & 64 & 263 & $\mathbf{7 5}$ \\
$\mathbf{7 0}$ & & 54 & 92 & & 63 & 260 & $\mathbf{7 0}$ \\
$\mathbf{6 5}$ & 28 & 53 & 91 & 23 & 62 & 257 & $\mathbf{6 5}$ \\
\hline $\mathbf{6 0}$ & & 52 & 89 & & 61 & 254 & $\mathbf{6 0}$ \\
$\mathbf{5 5}$ & 27 & 51 & 88 & 22 & 60 & 251 & $\mathbf{5 5}$ \\
$\mathbf{5 0}$ & 16 & 50 & 87 & & & 248 & $\mathbf{5 0}$ \\
$\mathbf{4 5}$ & & 49 & 86 & & 59 & 242 & $\mathbf{4 5}$ \\
\hline $\mathbf{4 0}$ & 25 & & 85 & 21 & 58 & 238 & $\mathbf{4 0}$ \\
$\mathbf{3 5}$ & 24 & 48 & 83 & & 56 & 233 & $\mathbf{3 5}$ \\
$\mathbf{3 0}$ & 23 & 47 & 82 & 20 & 55 & 227 & $\mathbf{3 0}$ \\
$\mathbf{2 5}$ & & 46 & 80 & & 53 & 224 & $\mathbf{2 5}$ \\
\hline $\mathbf{2 0}$ & 22 & 44 & 78 & 19 & 52 & 218 & $\mathbf{2 0}$ \\
$\mathbf{1 5}$ & 20 & 43 & 75 & 18 & 51 & 213 & $\mathbf{1 5}$ \\
$\mathbf{1 0}$ & 19 & 41 & 72 & 17 & 49 & 207 & $\mathbf{1 0}$ \\
$\mathbf{5}$ & 14 & 38 & 68 & 16 & 47 & 195 & $\mathbf{5}$ \\
\hline Media & $\mathbf{2 5 . 8 4}$ & $\mathbf{5 0 . 0 2}$ & $\mathbf{8 6 . 6 2}$ & $\mathbf{2 1 . 7 7}$ & $\mathbf{5 3 . 9 9}$ & $\mathbf{2 4 3 . 6 4}$ & Media \\
\hline $\mathbf{D . E}$ & $\mathbf{4 . 8 8}$ & $\mathbf{6 . 8 6}$ & $\mathbf{1 0 . 8 7}$ & $\mathbf{3 . 6 8}$ & $\mathbf{8 . 2 7}$ & $\mathbf{2 8 . 2 4}$ & D.E. \\
\hline $\mathbf{N}$ & & & $\mathbf{4 8 9}$ & & & $\mathbf{N}$ \\
\hline
\end{tabular}

\section{BIBLIOGRAFIA}

Alarcón, R. (1991). Métodos y diseños de Investigación del Comportamiento. Lima: U.P.C.H., Fondo Editorial.

Anastasi, A. Urbina, S. (1998). Test Psicológicos. México: Editorial Prentice Hall

Ashforth, B.E. \& Humphrey, R.H. (1995). Emotion in the workplace: A reappraisal. Human Relations, 48(2), 97-125.

Brockert, S. \& Braun G. (1997). Los tests de la Inteligencia Emocional. Barcelona: Ediciones Robinbook.

Brown, F. (1980). Principios de la Medición en Psicología y Educación. México:

Edit. El Manual Moderno. 
Cooper, R.\& Sawaf, A. (1997) La Inteligencia Emocional aplicada al liderazgo y a las organizaciones. Barcelona: Grupo Editorial Norma.

García Vega, L. (1998). 100 años de la ley del Efecto a la Inteligencia Emocional.

Gardner, H. (1993). Estructuras de la mente: La teoría de las Múltiples inteligencias. México: Fondo de Cultura Económica.

Goleman, D (1996). Inteligencia Emocional. Barcelona: Editorial Kairos.

Goleman, D. (1997). La Inteligencia Emocional de los niños. Buenos Aires: Javier Vergara Editor S. A. .

Hernández, Fernández, Baptista. (1998). Metodología de la investigación. México:

Mc. Graw-Hill.

Kerlinger, F. (1988) Investigación del comportamiento. México: Mac Graw-Hill

Marín, G. (1986). Consideraciones metodológicas básicas para conducir investigaciones Psicológicas en América Latina. Acta Psiquiátrica y Psicológica de América de Latina Vol. 32, Pgs. 183-192.

Mestre, J. M., Carreras M. y Guil, M (1998). Una aproximación a la evaluación de la Inteligencia Emocional como constructo teórico.

Mayer, J. D. \& Salovey, P. (1993). The Intelligence of emotional intelligence. Intelligence. Vol 17, pp. 433-442.

Mayer, J. D. \& Salovey, P. (J997). Whay is emotional intelligence. In Salovey P, \& Sluyter (Eds.). Emotional development literacy and Emotional Intelligence: implications for educator. New York: Basic Books ..

Muñiz, J. (1996). Psicometría. Madrid: Ed. Universitas S. A.

Salovey, P. \& Mayer, J.D. (1990). Emotional intelligence. Imagination, Cognition, and Personality, 9, 185-211.

Ruisel, I. (1992). Social Intelligence. Conception and methodological problems. Studia Psichologica, 34, (4-5),281-296.

Sánchez, C. H. y Reyes, M. C. (1998). Metodología y Diseños en la Investigación Científica: Aplicados a la Psicología, Educación y Ciencias Sociales. Lima: Editorial Mantaro.

Schwab, D.P. (1980). Construct validity in organization behavior. In B.M. Staw \& L.L. Cummings (Eds.), Research in organizational behavior, Vol. 2. Greenwich, CT: JAI Press.

Siegel, S. y Castellan N. (1995). Estadística No paramétrica: Aplicada a las ciencias de la conducta. México: Editorial Trillas.

Vitor G. (1998) Inteligencia Emocional e a Escola de Educacao Emocional, Brasilia:

Escola de Educacao Emocional

Williams, W.M. \& Sternberg, RJ. (1988). Group intelligence: Why some groups are better than others. Intelligence, 12,351-377.

Young, C (1996). Measuring a psychological construct. Organizational Behavior. 20, 250-279. 